\title{
Beating the channel capacity limit for linear photonic superdense coding
}

\author{
JULIO T. BARREIRO*, TZU-CHIEH WEI† AND PAUL G. KWIAT \\ Department of Physics, University of Illinois at Urbana-Champaign, Urbana, Illinois 61801-3080, USA \\ † Present address: Institute for Quantum Computing and Department of Physics and Astronomy, University of Waterloo, Waterloo ON N2L 3G1, Canada \\ *e-mail: juliob@uiuc.edu
}

Dense coding is arguably the protocol that launched the field of quantum communication ${ }^{1}$. Today, however, more than a decade after its initial experimental realization ${ }^{2}$, the channel capacity remains fundamentally limited as conceived for photons using linear elements. Bob can only send to Alice three of four potential messages owing to the impossibility of carrying out the deterministic discrimination of all four Bell states with linear optics ${ }^{3,4}$, reducing the attainable channel capacity from 2 to $\log _{2} 3 \approx 1.585$ bits. However, entanglement in an extra degree of freedom enables the complete and deterministic discrimination of all Bell states ${ }^{5-7}$. Using pairs of photons simultaneously entangled in spin and orbital angular momentum $^{8,9}$, we demonstrate the quantum advantage of the ancillary entanglement. In particular, we describe a dense-coding experiment with the largest reported channel capacity and, to our knowledge, the first to break the conventional linear-optics threshold. Our encoding is suited for quantum communication without alignment ${ }^{10}$ and satellite communication.

The first realization of quantum dense coding was optical, using pairs of photons entangled in polarization ${ }^{2}$. Dense coding has since been realized in various physical systems and broadened theoretically to include high-dimension quantum states with multiparties $^{11}$, and even coding of quantum states ${ }^{12}$. The protocol extension to continuous variables ${ }^{13,14}$ has also been experimentally explored optically, using superimposed squeezed beams ${ }^{15}$. Other physical approaches include a simulation in nuclear magnetic resonance with temporal averaging ${ }^{16}$, and an implementation with atomic qubits on demand without postselection ${ }^{17}$. However, photons remain the optimal carriers of information given their resilience to decoherence and ease of creation and transportation.

Quantum dense coding was conceived ${ }^{1}$ such that Bob could communicate 2 bits of classical information to Alice with the transmission of a single qubit, as follows. Initially, each party holds one spin-1/2 particle of a maximally entangled pair, such as one of the four Bell states. Bob then encodes his 2-bit message by applying one of four unitary operations on his particle, which he then transmits to Alice. Finally, Alice decodes the 2-bit message by discriminating the Bell state of the pair.

Alice's decoding step, deterministically resolving the four Bell states, is known as Bell-state analysis (BSA). Although in principle attainable with nonlinear interactions, such BSA with photons is very difficult to achieve with present technology, yielding extremely low efficiencies and low discrimination fidelities ${ }^{18}$. Therefore, current fundamental studies and technological developments demand the use of linear optics. However, for quantum communication, standard BSA with linear optics is fundamentally impossible $e^{3,4}$. At best, only two Bell states can be discriminated; for quantum communication, the other two are considered together for a three-message encoding. Consequently, the maximum channel capacity of this conventional optical dense coding is $\log _{2} 3 \approx 1.585$ bits. Although there are probabilistic approaches that can distinguish all four Bell states (which would be necessary to achieve the fundamental channel capacity of 2), these are at best successful $50 \%$ of the time ${ }^{19}$, so have a net channel capacity of at most 1 per photon.

Entanglement in an extra degree of freedom (DOF) of the pair, hyperentanglement ${ }^{20}$, enables full BSA with linear optics ${ }^{5,6}$. In this case, because Bob only encodes information in one DOF (the auxiliary DOF is unchanged), a dense-coding protocol proceeds under the same encoding conditions as in the original proposal $^{1}$. Although hyperentanglement-assisted BSA (HBSA) on polarization states has been reported with ancillas entangled in energy-time ${ }^{6}$ and linear momentum ${ }^{7}$, no advantage for quantum information or fundamental physics was shown; experiments thus far have been limited to a channel capacity of less than $1.18(3)$ bits $^{6}$, substantially less than is possible even without hyperentangled resources.

Using pairs of photons entangled in their spin and orbital angular momentum (OAM) in a HBSA with high stability and high detection fidelity, we realize a dense-coding experiment with a channel capacity that exceeds the threshold to beat conventional linear-optics schemes. In our scheme, Alice and Bob are provided with pairs of photons simultaneously entangled in their spin and \pm 1 -OAM in a state of the form

$$
\frac{1}{2}(|H H\rangle+|V V\rangle) \otimes(|\circlearrowleft \circlearrowright\rangle+|\circlearrowright \circlearrowleft\rangle)
$$

Here, $H(V)$ represents the horizontal (vertical) photon polarization and $\circlearrowleft(\circlearrowright)$ represents the paraxial spatial modes (Laguerre-Gauss) carrying $+\hbar(-\hbar)$ units of $\mathrm{OAM}^{21}$. Bob encodes his message by applying one of four unitary operations on the spin of his photon of this hyperentangled pair: (1) the identity, (2) $V \rightarrow-V$, (3) $H \leftrightarrow V$ or (4) $V \rightarrow-V$ and $H \leftrightarrow V$. Such operations transform the state in equation (1) into

$$
\Phi_{\text {spin }}^{ \pm} \otimes \Psi_{\text {orbit }}^{+}, \quad \text { and } \quad \Psi_{\text {spin }}^{ \pm} \otimes \Psi_{\text {orbit }}^{+}
$$




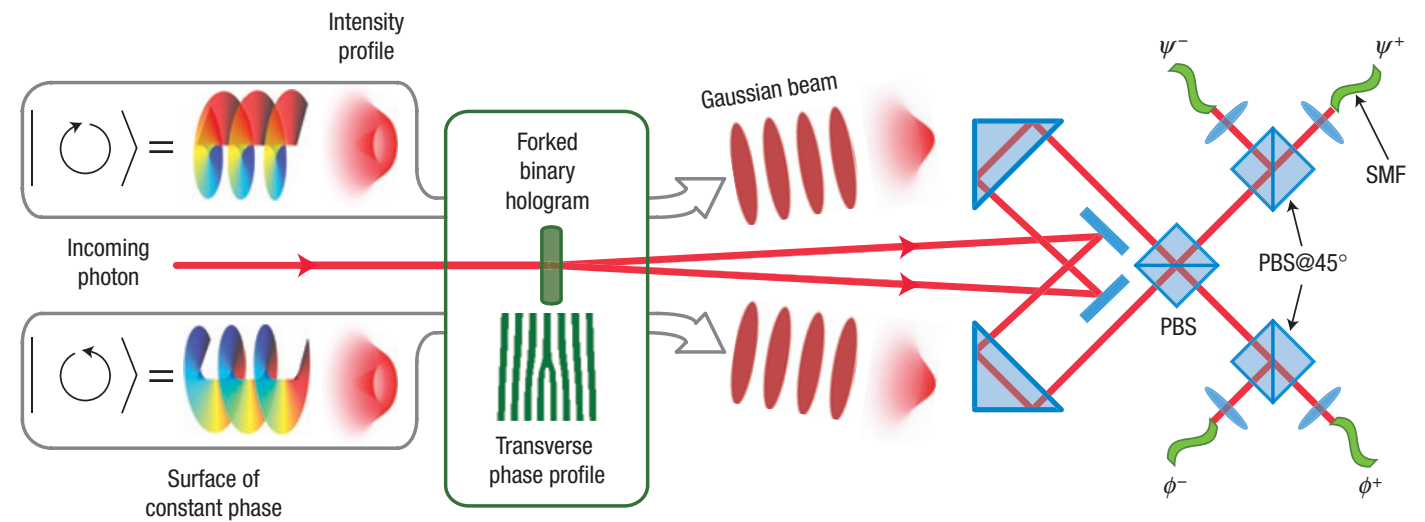

Figure 1 Spin-orbit Bell-state analyser. A photon in a spin-orbit Bell state incident from the left is first split according to its $\pm 1-0 \mathrm{AM}$ content; its $\pm 1-0 \mathrm{AM}$ components are converted to 0-OAM and combined on a PBS for a spin-controlled orbit-CNOT gate. The photon is then filtered by a single-mode fibre (SMF) and finally routed to a unique detector (photon-counting avalanche photodiode).

where the spin and orbit Bell states are defined as

$$
\begin{gathered}
\Phi_{\text {spin }}^{ \pm} \equiv(|H H\rangle \pm|V V\rangle) / \sqrt{2}, \\
\Psi_{\text {spin }}^{ \pm} \equiv(|H V\rangle \pm|V H\rangle) / \sqrt{2}, \\
\Psi_{\text {orbit }}^{+} \equiv(|\circlearrowleft \circlearrowright\rangle+|\circlearrowright \circlearrowleft\rangle) / \sqrt{2} .
\end{gathered}
$$

We designed a HBSA scheme (inspired by ref. 22) enabling Alice to discriminate the four states in equation (2). In this scheme, the polarization BSA relies on the observation that the states resulting from Bob's encoding can be rewritten as superpositions of the single-photon Bell states of spin and OAM, or spin-orbit Bell states:

$$
\begin{aligned}
\phi^{ \pm} & \equiv \frac{1}{\sqrt{2}}(|H \circlearrowleft\rangle \pm|V \circlearrowright\rangle), \\
\psi^{ \pm} & \equiv \frac{1}{\sqrt{2}}(|H \circlearrowright\rangle \pm|V \circlearrowleft\rangle) .
\end{aligned}
$$

On this basis, the states Alice analyses have the form

$$
\begin{aligned}
\Phi_{\text {spin }}^{ \pm} \otimes \Psi_{\text {orbit }}^{+}= & \frac{1}{4}\left(\phi_{1}^{+} \otimes \psi_{2}^{\mp}+\phi_{1}^{-} \otimes \psi_{2}^{ \pm}\right. \\
& \left.+\psi_{1}^{+} \otimes \phi_{2}^{ \pm}+\psi_{1}^{-} \otimes \phi_{2}^{\mp}\right), \\
\Psi_{\text {spin }}^{ \pm} \otimes \Psi_{\text {orbit }}^{+}= & \frac{1}{4}\left(\phi_{1}^{+} \otimes \phi_{2}^{\mp}+\phi_{1}^{-} \otimes \phi_{2}^{ \pm}\right. \\
& \left.+\psi_{1}^{+} \otimes \psi_{2}^{ \pm}+\psi_{1}^{-} \otimes \psi_{2}^{\mp}\right) .
\end{aligned}
$$

This arrangement shows that each hyperentangled state is a unique superposition of four of the sixteen possible combinations of twophoton spin-orbit Bell states. Therefore, Alice can decode Bob's message by carrying out spin-orbit BSA locally on each photon.

We implement the spin-orbit BSA with a novel interferometric apparatus consisting of a $\pm 1-\mathrm{OAM}$ splitter and polarizing beam splitters (PBSs), as shown in Fig. 1. The first splitter combines the action of a binary plane-wave phase grating ${ }^{21}$ and single-mode fibres. The grating transforms an incoming photon in the state $|\circlearrowleft\rangle(|\circlearrowright\rangle)$ into a gaussian beam with no OAM in the $+1(-1)$ diffraction order (for a splitter that preserves the photon's OAM, see ref. 21). Subsequently filtering the first diffraction orders with

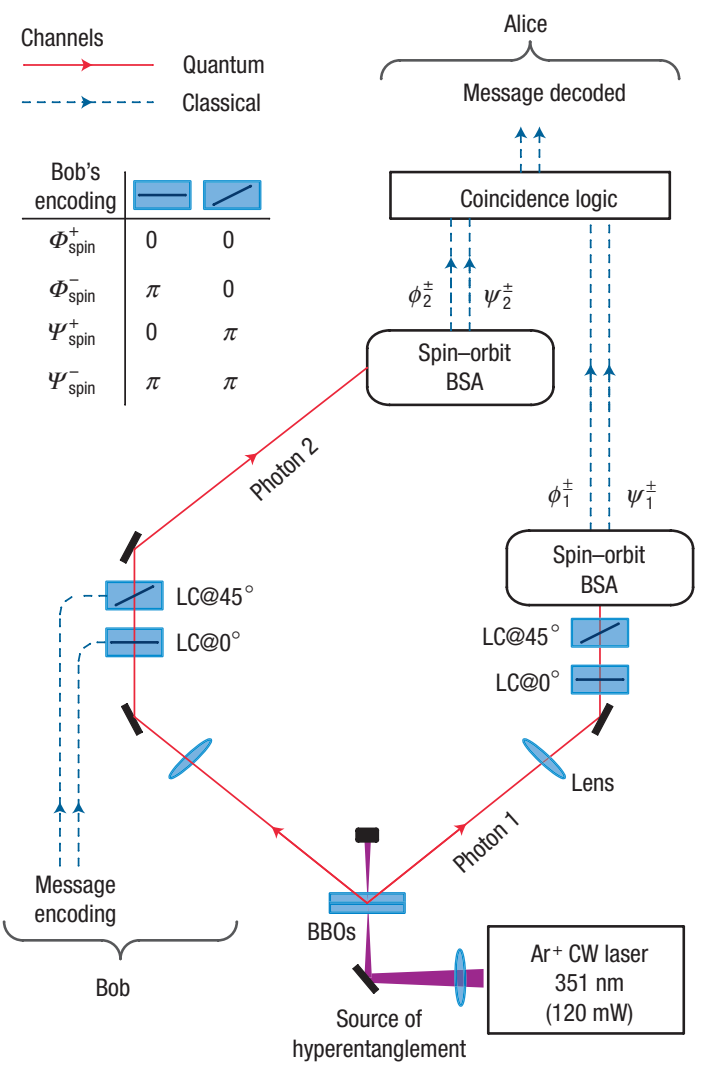

Figure 2 Experimental set-up for dense coding with spin-orbit encoded photons. Acting on photon 2 of a hyperentangled pair, Bob encodes his message by using the liquid crystals (LCs) to apply the phases indicated in the table; at the same time (or earlier) Alice carries out spin-orbit BSA on photon 1. Later-the upward direction suggests time progression-Alice uses a spin-orbit BSA on photon 2, and the result from the measurement on photon 1, to decode Bob's message. The liquid crystals on the path of photon 1 applied no phase during the dense-coding experiment, but were used along with Bob's liquid crystals to characterize the polarization states of the hyperentangled source by quantum state tomography. The liquid-crystal optic axes are perpendicular to the incident beams; LC@45 (LC@0 $)$ is oriented at $45^{\circ}\left(0^{\circ}\right)$ from the horizontal polarization direction. BB0s: $\beta$-barium borate nonlinear crystals; CW: continuous-wave. 


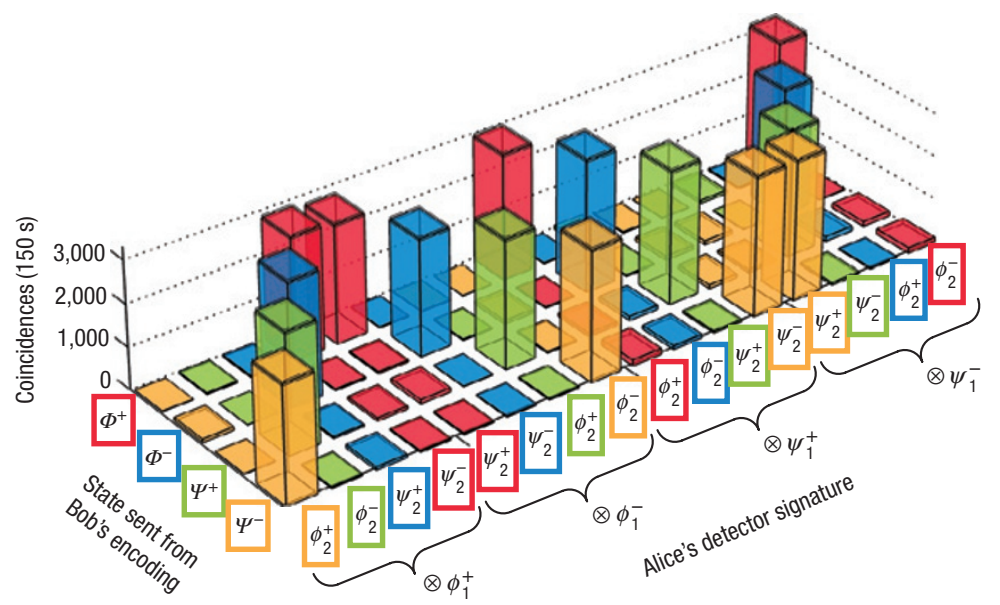

Figure 3 Experimental results of hyperentanglement-assisted dense coding. Coincidence counts detected by Alice's HBSA for each message (state) sent by Bob. The error bars (shown as additional squares at the top of each column) represent \pm 1 standard deviations, deduced from poissonian counting statistics. The state-discrimination SNRs, which compare the sum of the four rates corresponding to the actual state to the sum of the other twelve registered rates, are SNR $_{\Phi^{+}}=19.9(8)$, SNR $_{\Phi^{-}}=27(1)$, $\operatorname{SNR}_{\Psi^{+}}=13.7(5)$ and $\operatorname{SNR}_{\Psi^{-}}=16.4(6)$.

single-mode fibres, we effectively split an incoming photon into its \pm 1 -OAM components. By merging these diffraction orders on a PBS, we carry out a spin-controlled NOT gate over the photon OAM. In Fig. 1, the states $\psi^{ \pm}\left(\phi^{ \pm}\right)$exit on the top (bottom) output port of the PBS. Followed by measurements in the diagonal basis, shown in Fig. 1 as PBS@ $45^{\circ}$, the desired measurement in the single-photon Bell-state basis is accomplished. Further birefringent elements make this device a universal unitary gate for single-photon two-qubit states, in analogy with the device for polarization-linear momentum states in ref. 23.

Each step in the dense-coding protocol corresponds to a distinct experimental stage in Fig. 2: a hyperentanglement source, Bob's encoding components and Alice's HBSA. The hyperentanglement source is realized via spontaneous parametric downconversion in a pair of nonlinear crystals (see the Methods section). The generated photon pairs are entangled in polarization, OAM and emission time ${ }^{9}$. In particular, we use a subspace of the produced states that was shown to have a state overlap or fidelity of $97 \%$ with the state in equation (1). Next, Bob encodes his message in the polarization state by applying birefringent phase shifts with a pair of liquid crystals, as shown in Fig. 2. Finally, Alice carries out HBSA using two of the spin-orbit Bell-state analysers shown in Fig. 1, one for each photon (see the Methods section).

We characterize our dense-coding implementation by switching between the four states for equal intervals, and measuring all output states of the HBSA. The results of these measurements are coincidence counts for each input state, as shown in Fig. 3. From this data, we can determine the conditional detection probabilities that Alice detects each message $\Phi^{ \pm}$and $\Psi^{ \pm}$that Bob sent, for example, the message $\Phi^{+}$. The probabilities shown in Fig. 4 were calculated by comparing the sum of the four rates corresponding to each detected message over the sum of all sixteen rates for the sent message. The average probability of success was $94.8(2) \%$ (all reported errors from Monte Carlo simulations).

A better figure of merit for a quantum dense-coding implementation is the channel capacity, because it characterizes the exponential growth of the maximum number of distinguishable signals for a given number of uses of the channel (see the Methods section). From the conditional detection probabilities, we obtain a channel capacity of $1.630(6)$ bits with a probability of sending each state of $P\left(\Phi^{+}\right)=0.26, P\left(\Phi^{-}\right)=0.26, P\left(\Psi^{+}\right)=0.24$ and $P\left(\Psi^{-}\right)=0.24$. This exceeds the 1.585 channel capacity threshold for conventional linear-optics implementations. The channel capacity drifted by no more than one standard deviation between experimental runs, demonstrating the high stability of the implementation.

The experimental channel capacity is nevertheless smaller than the maximum attainable (2 bits), owing to imperfections in the alignment, input states and components. By characterizing each imperfection and modelling the gates and measurement, we estimated their effect on the channel capacity (see Supplementary Information, Part II). Considering all mentioned imperfections (see the Methods section) and their spread in a Monte Carlo simulation, the predicted channel capacity of 1.64(2) bits agrees with the measured channel capacity of $1.630(6)$ bits. The polarization and spatial-mode states can be improved by spatially compensating the angle-dependent phase $\mathrm{e}^{24}$, using a forked hologram with a smaller diffraction angle to decrease wavelength dispersion (a potential source of alignment imbalances), and obtaining crystals with a smaller wedge. The deleterious effect of the PBS crosstalk can be reduced by adding extra phase-compensation plates inside the interferometers, and can potentially be eliminated altogether by adding appropriate birefringent beam displacers after each PBS.

Above, Bob encoded two qubits in the form of spin-orbit Bell states by acting only on the spin DOF. However, more generally he could also apply one of four unitaries in the \pm 1 -OAM subspace and encode four qubits. The state of the pair of photons then becomes a product of Bell states, 16 in total. In principle, if Alice could discriminate all of these 'hyper-Bell' states, up to 4 bits could be transmitted per photon. We have investigated the limits for unambiguously distinguishing these Bell-like states, and have found that the optimal one-shot discrimination scheme is to group the 16 states into 7 distinguishable classes ${ }^{25}$. The optimal analysis can be achieved by the Kwiat-Weinfurter scheme ${ }^{5}$, with photonnumber resolving detectors, giving a maximum channel capacity of $\log _{2} 7 \approx 2.81$ bits. If we modify the present scheme, we can also implement an unambiguous discrimination of all 16 Bell states with two identical copies ${ }^{25}$.

In conclusion, we have beaten a fundamental limit on the channel capacity for standard dense coding using only linear optics. A number of features make our HBSA efficient and reliable. First, 

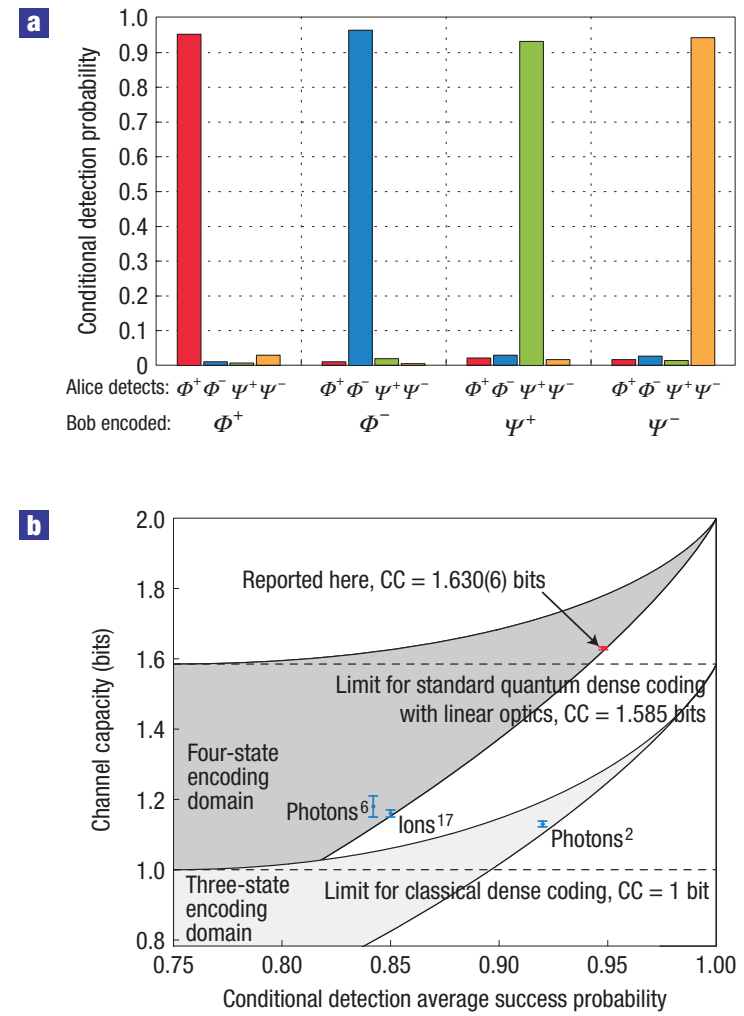

Figure 4 Conditional detection probabilities beating the channel capacity limit for standard dense coding with linear optics. a, Given that Bob encoded the four states indicated, Alice infers the state transmitted with the probabilites shown (calculated from data in Fig. 3). Her average success probability is $94.8(2) \%$. The uncertainty in each probability is less than $0.2 \%$. These results imply a channel capacity (CC) of 1.630(6) bits, above the standard linear-optics limit of 1.585. b. Experimentally reported channel capacities as a function of their conditional detection average success probability. The error bars represent the statistical error of \pm 1 standard deviations. The domains of achievable channel capacity for both three- and four-state encodings are shown for reference (see Supplementary Information, Part I).

hyperentanglement offers advantages in the source, logic gates and detection side. Quantum logic between qubits encoded on different DOFs is much more easily implemented than when using different photons ${ }^{26,27}$. From the source side, more quantum information is available per photon, particularly with the energy-time and spatialmode DOF (for example, ref. 28). In the detection side, compared with multiphoton approaches, higher efficiency is achieved because only one pair of photons is detected. Second, because our HBSA requires only local measurements, Alice can measure one of the photons and store the classical result of her measurement until Bob sends his photon (she does not require a quantum memory). Finally, the photon's polarization and \pm 1 -OAM constitute a robust encoding as they enable quantum communication without alignment ${ }^{10}$ as well as other landmark advances for quantum information ${ }^{8}$. Furthermore, by using paraxial beams as the ancillary DOF, the scheme is free of tight source-to-detector requirements such as interferometric stability ${ }^{7}$ or perfect indistiguishability for Hong-Ou-Mandel interference ${ }^{6}$. However, OAM single-photon and entangled states easily decohere by atmospheric turbulence ${ }^{29,30}$, limiting their likely communication applications to satellite-tosatellite transmissions.
METHODS

The hyperentanglement source is realized by directing $120 \mathrm{~mW}$ of $351 \mathrm{~nm}$ light from a continuous-wave $\mathrm{Ar}^{+}$laser into two contiguous $\beta$-barium borate nonlinear crystals with optic axes aligned in perpendicular planes ${ }^{9}$. Type-I degenerate $702 \mathrm{~nm}$ photons in a $3.0^{\circ}$ half-opening angle cone are produced by phase-matching each $0.6-\mathrm{mm}$-thick crystal. In the spin and \pm 1 OAM subspace, a two-fold coincidence rate of five detected pairs per second is determined by a $10 \mathrm{~ns}$ coincidence window and interference filters with $\Delta \lambda_{\mathrm{FWHM}}=5 \mathrm{~nm}$.

In our HBSA implementation, each PBS@45 and its two outputs in the spin-orbit BSA (Fig. 1) were replaced by a dichroic polarizer oriented at either $45^{\circ}$ or $-45^{\circ}$ and a single output; Alice's HBSA thus acquires all spin-orbit BSA outputs from four polarizer settings. With the continuouswave source, Alice cycles through the four polarizer settings, and for each polarizer setting Bob encodes the four messages, each for $150 \mathrm{~s}$. During the measurement, no active stabilization or realignment was done on the source, spin-orbit BSA interferometers or coupling optics. The HBSA polarizers and liquid crystals were quickly set with computer-controlled rotation stages and liquid-crystal controllers.

The wavelength-dependent voltage applied to each liquid crystal was independently calibrated to produce a birefringent phase difference of 0 or $\pi$ with a diode laser operated at $699 \mathrm{~nm}$ (Hitachi HL-6738MG, driven at $140 \mathrm{~mA}$ and $80^{\circ} \mathrm{C}$ ); the same laser was used to align the \pm 1 -OAM splitter. The binary forked holograms were silver-halide emulsion gratings with 33\% diffraction efficiency into the first order (more efficient schemes are described in ref. 21). The same holographic plate included spatial-mode tomography patterns, which in conjunction with the liquid crystals were used for state reconstruction ${ }^{9}$. The spurious phase on reflection on the PBS was compensated with a waveplate in each output port of the PBS for both spin-orbit Bell-state analysers. The state-discrimination signal-to-noise ratio (SNR) varied between states owing to mode-coupling imbalance in the spin-orbit BSA, PBS crosstalk and slight offsets in the liquid crystal calibrations.

We characterized the source polarization state $\Phi_{\text {spin }}^{-}$by quantum state tomography in the $|\circlearrowleft \circlearrowright\rangle$ and $|\circlearrowright \circlearrowleft\rangle$ OAM subspaces ${ }^{9}$ (using liquid crystals shown in Fig. 2 and PBSs of each spin-orbit BSA shown in Fig. 1). Considering all combinations of signature detectors, we measured an average degree of entanglement, or tangle, of $T=96.7(8) \%$ and a mixture or linear entropy of $S_{\mathrm{L}}=2.0(4) \%$. If such high-quality polarization states were exactly the same for each combination of signature detectors, the decrease in the channel capacity would be only 0.006 bits. However, small differences in the coupled state between each combination of detectors (expressed above as uncertainty) result in a channel capacity decrease of 0.09(2) (see Supplementary Information, Part II). The OAM state was also tomographically reconstructed in the $|H H\rangle$ and $|V V\rangle$ polarization subspaces ${ }^{9}$, measuring an average $T=91(3) \%$ and $S_{\mathrm{L}}=6(2) \%$, yielding a channel capacity decrease of $0.20(3)$ bits. The PBS crosstalk $(0.5 \%$ for $H, 1.0 \%$ for $V)$ further decreases the channel capacity by 0.10 (1) bits. Finally, accidental coincidences ( 5 in 150 s) reduce channel capacity by 0.02 bits.

\section{CHANNEL CAPACITY}

The capacity of a noisy channel is given by $\mathrm{CC}=\max _{p(x)} H(X: Y)$, where $x$ is in the space of signals that can be transmitted $X, H(X: Y)$ is the mutual information of $X$ and the space of received signals $Y$ and the maximum is taken over all input distributions $p(x) . H(X: Y)$ is a function of $p(x)$ and the conditional detection distribution $p(y \mid x)$ of receiving $y$ given that $x$ was sent:

$$
H(X: Y)=\sum_{y \in Y} \sum_{x \in X} p(x) p(y \mid x) \log \frac{p(y \mid x)}{\sum_{x \in X} p(y \mid x) p(x)} .
$$

In our experiment, a uniform probability of transmission gives a mutual information of 1.629(6) bits, negligibly smaller than the channel capacity owing to the nearly balanced conditional probabilities, that is, there is little to be gained by sending some states more frequently.

Received 18 June 2007; accepted 7 February 2008; published 23 March 2008.

\section{References}

1. Bennett, C. H. \& Wiesner, S. J. Communication via one- and two-particle operators on Einstein-Podolsky-Rosen states. Phys. Rev. Lett. 69, 2881-2884 (1992).

2. Mattle, K. et al. Dense coding in experimental quantum communication. Phys. Rev. Lett. 76, 4656-4659 (1996).

3. Vaidman, L. \& Yoran, N. Methods for reliable teleportation. Phys. Rev. A 59, 116-125 (1999). 
4. Lütkenhaus, N., Calsamiglia, J. \& Suominen, K. A. Bell measurements for teleportation. Phys. Rev. A 59, 3295-3300 (1999)

5. Kwiat, P. G. \& Weinfurter, H. Embedded Bell-state analysis. Phys. Rev. A 58, R2623-R2626 (1998),

6. Schuck, C., Huber, G., Kurtsiefer, C. \& Weinfurter, H. Complete deterministic linear optics Bell state analysis. Phys. Rev. Lett. 96, 190501 (2006)

7. Barbieri, M., Vallone, G., Mataloni, P. \& Martini, F. D. Complete and deterministic discrimination of polarization Bell states assisted by momentum entanglement. Phys. Rev. A 75, 042317 (2007).

8. Molina-Terriza, G., Torres, J. P. \& Torner, L. Twisted photons. Nature Phys. 3, 305-310 (2007)

9. Barreiro, J. T., Langford, N. K., Peters, N. A. \& Kwiat, P. G. Generation of hyperentangled photon pairs. Phys. Rev. Lett. 95, 260501 (2005).

10. Aolita, L. \& Walborn, S. P. Quantum communication without alignment using multiple-qubit single-photon states. Phys. Rev. Lett. 98, 100501 (2007).

11. Liu, X. S., Long, G. L., Tong, D. M. \& Li, F. General scheme for superdense coding between multiparties. Phys. Rev. A 65, 022304 (2002).

12. Harrow, A., Hayden, P. \& Leung, D. Superdense coding of quantum states. Phys. Rev. Lett. 92, 187901 (2004).

13. Braunstein, S. L. \& Kimble, H. J. Dense coding for continuous variables. Phys. Rev. A 61 , 042302 (2000).

14. Ban, M. Quantum dense coding via a two-mode squeezed-vacuum state. J. Opt. B 1, L9 (1999).

15. Li, X. et al. Quantum dense coding exploiting a bright Einstein-Podolsky-Rosen beam. Phys. Rev Lett. 88, 047904 (2002)

16. Fang, X., Zhu, X., Feng, M., Mao, X. \& Du, F. Experimental implementation of dense coding using nuclear magnetic resonance. Phys. Rev. A 61, 022307 (2000).

17. Schaetz, T. et al. Quantum dense coding with atomic qubits. Phys. Rev. Lett. 93, 040505 (2004).

18. Kim, Y.-H., Kulik, S. P. \& Shih, Y. Quantum teleportation of a polarization state with a complete Bell state measurement. Phys. Rev. Lett. 86, 1370-1373 (2001).

19. Calsamiglia, J. \& Lütkenhaus, N. Maximum efficiency of a linear-optical Bell-state analyzer. Appl. Phys. B 72, 67-71 (1999)

20. Kwiat, P. G. Hyper-entangled states. J. Mod. Opt. 44, 2173-2184 (1997).
21. Allen, L., Barnett, S. M. \& Padgett, M. J. (eds) Optical Angular Momentum (Institute of Physics Publishing, Bristol, 2003)

22. Walborn, S. P., Pádua, S. \& Monken, C. H. Hyperentanglement-assisted Bell-state analysis. Phys. Rev. A 68, 042313 (2003).

23. Englert, B.-G., Kurtsiefer, C. \& Weinfurter, H. Universal unitary gate for single-photon two-qubit states. Phys. Rev. A 63, 032303 (2001).

24. Altepeter, J. B., Jeffrey, E. R. \& Kwiat, P. G. Phase-compensated ultra-bright source of entangled photons. Opt. Express 13, 8951-8959 (2005).

25. Wei, T.-C., Barreiro, J. T. \& Kwiat, P. G. Hyperentangled Bell-state analysis. Phys. Rev. A 75, 060305(R) (2007)

26. Cerf, N. J., Adami, C. \& Kwiat, P. G. Optical simulation of quantum logic. Phys. Rev. A 57, R1477-R1480 (1998)

27. Fiorentino, M. \& Wong, F. N. C. Deterministic controlled-not gate for single-photon two-qubit quantum logic. Phys. Rev. Lett. 93, 070502 (2004).

28. Ali-Khan, I., Broadbent, C. J. \& Howell, J. C. Large-alphabet quantum key distribution using energy-time entangled bipartite states. Phys. Rev. Lett. 98, 060503 (2007).

29. Paterson, C. Atmospheric turbulence and orbital angular momentum of single photons for optical communication. Phys. Rev. Lett. 94, 153901 (2005).

30. Smith, B. J. \& Raymer, M. G. Two-photon wave mechanics. Phys. Rev. A 74, 062104 (2006).

Acknowledgements

We thank N. Peters and N. Langford for helpful discussions and R. Hillmer for assistance in hologram fabrication. This work was jointly supported by the DTO/ARO-sponsored MURI Center for Photonic Quantum Information Systems and the DTO/IARPA-sponsored Advanced Quantum Communication grant. J.T.B. acknowledges support from CONACYT-México.

Correspondence and requests for materials should be addressed to J.T.B.

Supplementary Information accompanies this paper on www.nature.com/naturephysics.

Reprints and permission information is available online at http://npg.nature.com/reprintsandpermissions/ 